\title{
Del empleo al post-empleo: O de la plasticidad de la psicología en la producción de la subjetividad laboral
}

\author{
Do emprego para o pós-emprego: Ou da plasticidade da psicologia na \\ produção de subjetividade laboral \\ From employment to post-employment: On the plasticity of psychology as \\ regards the production of workers' subjectivity
}

\author{
Hernan Camilo PULIDO-MARTÍNEZ \\ Pontificia Universidad Javeriana, Bogotá, Colômbia
}

Resumen En este artículo se considera la re-organización del "complejo psi", en relación a las transformaciones que ocurren contemporáneamente en el mundo del trabajo. Para esto se hace un recorrido examinando los cambios en el lugar y las operaciones de la psicología con respecto a las formas sociales empleo y post-empleo. Se argumenta que el papel de la psicología en relación a la producción de la subjetividad laboral se ha ido transformando en medio a los cambios que ávidamente han ido socavando lo que ahora se conoce como el "buen trabajo". Estas mutaciones se caracterizan por el aumento acelerado de incertidumbres relacionadas con los distintos aspectos de las condiciones laborales tales como, la contratación, los horarios, los espacios laborales, y las relaciones interpersonales. Se concluye que, en este contexto, la psicología juega un papel preponderante para la producción de los nuevos sujetos que requiere la organización capitalista del trabajo.

Palabras-clave:

Subjetividad; empleo; post-empleo; psicología del trabajo; complejo psi.

Resumo Neste artigo, a reorganização do "complexo psi" é considerada em relação às transformações que estão ocorrendo no mundo do trabalho contemporâneo. Para isso, examinam-se as mudanças ocorridas com o ambiente e as operações da psicologia em relação às formas sociais de emprego e pós-emprego. Argumenta-se que o papel da psicologia em relação à produção da subjetividade laboral modifica-se em meio às transformações que, avidamente, vêm debilitando o que agora se conhece como o "bom trabalho". Essas mutações são caracterizadas pelo aumento acelerado das incertezas relacionadas com diferentes aspectos das condições de trabalho, como contratação, horários, espaços de trabalho e relações interpessoais. Conclui-se que, nesse contexto, a psicologia desempenha papel importante para a produção de novos sujeitos requeridos pela organização capitalista do trabalho. Palavras-chave:

Subjetividade; emprego; pós-emprego; psicologia do trabalho; complexo psi.

Abstract In this article the re-organization of the "psy complex" is considered as regards the contemporary transformations that are taking place in the world of work. Toward this end, a review of the changes in the place and operations of psychology with respect to the employment and post-employment social forms is conducted. It is argued that the role of psychology in the production of workers' subjectivity is transforming amidst the mutations that have been avidly debilitating what is at present known as "good work". These mutations are characterized by an increasing rate of incertitude in aspects such as work contracts, rosters, labor spaces, and interpersonal relationships. It is concluded that in this context, psychology plays a central role for the production of new subjects that the capitalist organization of work requires.

Keywords:

Subjectivity; employment; post-employment; work psychology; psy complex.

1 Endereço para contato: Pontificia Universidad Javeriana. Carrera 5 No 39-00, Ed Manuel Briceño of 203, Bogotá, Colômbia. Email: cpulido@javeriana.edu.co 
a primera premisa argumental que aquí se quiere sostener señala que la psicología del trabajo convencional o hegemónica, a la cual comúnmente se le conoce por apelativos tales como ocupacional, industrial y organizacional (Hollway, 1991), es muy pertinente para las condiciones de trabajo que el empleo representa. La segunda premisa que se postula señala que esta psicología es menos pertinente para la condición de post-empleo, situación en la cual otras psicologías están llegando a ocupar una posición hegemónica para entender e intervenir el trabajo. Se propone a lo largo de este artículo un análisis de esta tensión que surge entre las psicologías que se ocupan del mundo laboral. El examen de esta tensión se apoya, en el punto de vista adelantado en los estudios sociales de la psicología que hacen énfasis en que el reconocimiento social de este saber no necesariamente se rige por los parámetros que la cientificidad quisiera imponer (Gillespie, 1993; Hollway, 1991; Townley, 1994).

Específicamente con referencia a la relación entre psicología y trabajo, estos estudios han mostrado que desde que se empezó a entender al trabajador y a las organizaciones en términos psicológicos, como por ejemplo en la investigación que fue adelantada en la Hawthorne Electric Company en los Estados Unidos da América bajo la dirección de Mayo (1990), el conocimiento psicológico ha sufrido una serie de alteraciones que no dependen exclusivamente de la excelencia y rigurosidad con la que se lleve a cabo la investigación. Por el contrario, se ha demostrado que las relaciones establecidas entre diferentes instancias sociales que componen el complejo psi en el trabajo tales como, los investigadores de las facultades de psicología y de administración, las agremiaciones de profesionales, los sindicatos, las editoriales académicas, las importadoras de instrumentos psicológicos, las empresas, los gerentes, los psicólogos organizacionales y los mismos trabajadores, conforman una red que hace posible la tanto la producción de conocimiento psicológico sobre el trabajo, como la aplicación de ese conocimiento a los problemas laborales (Rose, 1985, 1999). En última instancia los estudios muestran como las relaciones que se establecen en la red determinan cual será la versión final, y por tanto oficial, de los resultados de investigación (Gillespie, 1993).

La pregunta inmediata que surge con referencia a esta situación resulta obvia: Si no es por la validez científica del conocimiento psicológico organizacional que este tiene tanta acogida y se aplica con tanta frecuencia en tantos lugares, ¿cuál o cuáles pueden ser la razones para que la psicología se expanda colonizando cada vez más los ámbitos laborales? Para responder esta pregunta los investigadores han señalado que la psicología del trabajo es un saber típicamente perteneciente a la modernidad y a la ciencia del trabajo que la acompañado (Meda, 1998), por tanto, su efectividad social está relacionada, a manera de ejemplo, con maneras de individualizar y al mismo tiempo homogenizar a la fuerza laboral. Es decir, a través de la psicologización se produce unas formas de subjetividad que están totalmente acorde con las necesidades que requiere la producción capitalista (Hollway, 1991; Pulido-Martínez \& Carvajal-Marín, 2014; Townley, 1994; Walkerdine, 2005).

En este artículo se explora entonces el proceso de psicologización del trabajo en el cambio que está acaeciendo entre dos formas de organizarlo. A lo largo del texto se muestra la plasticidad que tiene la psicología para adaptarse tanto a los cambios en la organización del trabajo, así por tanto a las racionalidades que rigen estos cambios.

\section{La psicología en el empleo}

Los estudios históricos sobre el desenvolvimiento de la relación entre la psicología y el trabajo muestran que esta relación siempre se ha propuesto como objetivo acercar las capacidades, aptitudes y conocimientos que el trabajador posee a la voluntad que este tiene para realizarlas (Viteles, 1932). A través de prometerle al mundo del trabajo que posee un conocimiento acerca de los contenidos que hay en la mente de los trabajadores, la psicología asumió para si la tarea de ganar la voluntad de quienes laboran. Obtuvo así la psicologia un lugar social, así como gano un puesto en concierto del conocimiento acerca del trabajo, en medio de las condiciones laborales asociadas a la forma social empleo (Gillespie, 1993; Hollway, 1991).

Bien sabían ya los psicólogos desde principios del siglo XX que sus propuestas estaban constituyendo un saber qué hacía eco del capitalismo, al punto que llegaron a afirmar que sin su conocimiento este sistema podría está bajo amenaza de desaparecer (Viteles, 1932). En este sentido, argumentaba Viteles ya en 1932 que el capitalismo no se preocupa por el trabajador puesto que a este se le concibe, en términos del Taylorismo, como la parte humana de la máquina que puede ser fácilmente reemplazable. Los psicólogos interesados en el trabajo dieron una vuelta a esta situación y formularon que sin psicología el capitalismo no podía sobrevivir 
puesto que solamente una preocupación por el bienestar, del trabajador, que ellos encarnaban, impediría que el capitalismo explotara (Viteles, 1932).

De esta manera, los psicólogos obtuvieron un lugar particular para su conocimiento en el mundo del trabajo en donde empezaron a mediar entre capacidades y voluntad, bienestar y enfermedad, y por supuesto entre democracia y autoritarismo en los lugares de trabajo. Adicionalmente, esta mediación se formuló a través de un proyecto general de felicidad en y por el trabajo que se construyó en la conjunción entre la psicología de las diferencias y la psicología humanista (Donzelot, 1981; Hollway, 1991; Rose, 1996; Triantafillou \& Moreira, 2005).

Básicamente, en términos psicológicos se propuso que la satisfacción con el trabajo es el centro del aumento de la productividad. La satisfacción con: las tareas que se realizan, la posición que se ocupa en el ámbito laboral y el tipo de administración que se lleva a cabo, es el resultado, casi exclusivo, de las percepciones que el individuo tiene de estos aspectos. Satisfacción y percepciones se constituyeron como centro de un proyecto de felicidad que iba a solventar los problemas relacionados con la manera de organizar el trabajo. Paradójicamente, el proyecto de felicidad psicológica dejó de lado las condiciones laborales, en su lugar se instituyó a la percepción del trabajador en relación con sus tareas, los jefes y los compañeros, como el foco de las dificultades que se presentaban en las organizaciones.

Por supuesto, como resultado del proyecto de felicidad, el "trabajador sentimental" surgió como figura de la subjetividad que permitía concebir a los seres humanos en el trabajo (Hollway, 1991). En esta figura la racionalidad del trabajador fue reemplazada por una emocionalidad que lo ubica en la posición de un sujeto infantil, es decir, en la posición de un "otro", del cual se puede afirmar se portaba como sujeto racional. Se constituyó así al administrador y al psicólogo del trabajo como figuras racionales que deben estar encargadas de guiar, controlar, educar, seleccionar, capacitar, y de manera ideal, de hacer feliz al trabajador. Entonces, se puede afirmar que desde su introducción en el mundo laboral, la psicología estaba destinada a realizar un cierta trivialización del mundo del trabajo en la medida en que se concentraba en la interioridad de ese sujeto por ella construido de manera tal que se podía llevar a cabo un efectivo "pastoreo" de los trabajadores, y al mismo, la misma psicologia permitía que se olvidaran las condiciones objetivas de trabajo al privilegiar las percepciones, los sentimientos, las motivaciones, la comunicación.

Sin embargo, aun poniendo de presente todos esos señalamientos y objeciones, el proyecto de felicidad psicológica en el trabajo tenía que tener algún tipo de resonancia en aquellos a los que iba dirigido de lo contrario no había podía alcanzar ninguna aceptación. Esta singularidad en la aceptación de la psicologia la proveían las condiciones laborales dentro de las cuales esta se pensó, diseñó y aplicó. En efecto, la forma social empleo proporcionó el marco para que fuese pertinente el proyecto psicológico de felicidad a través del cambio en las percepciones del trabajador. La construcción de una familia laboral, por ejemplo, que de cierta forma complementara, o reemplazara, los vínculos íntimos se constituyó en una estrategia importante que contribuiría a la satisfacción del trabajador. Así, este tipo de estrategias, solamente, podía tener resonancia en unas condiciones en donde el trabajador tenía vínculos a largo plazo, contratación permanente, horarios fijos, establecía unos roles específicos y construía una carrera.

Sobra anotar que la condición del empleo, resultado de una serie de reivindicaciones alcanzadas por los trabajadores, llevaba a que tanto el estado, como las organizaciones, compartieran una serie de responsabilidades sociales en medio de las cuales emergían las certidumbres laborales para que el proyecto de felicidad pudiese ser aplicado. Estas certidumbres asociadas a lo que ahora se conoce como "el buen trabajo" le proporcionan, también, al trabajador la libertad para estar por fuera del trabajo mismo, pues suministraban una serie de garantías que le permitían sobrevivir sin estar laborando cuando se presentaban casos de despido, o cuando sobrevenía la enfermedad y la vejez (Standing, 2010).

Se compuso entonces, una particular manera de concebir la subjetividad del trabajo en aquellos ámbitos laborales en donde se había establecido la forma social empleo. El trabajador sentimental se presentó como ese sujeto que se conduce a sí mismo, principalmente, por las relaciones sociales que establece con sus compañeros y con las figuras de autoridad, así como por la satisfacción que obtiene de su vinculación perceptual con las tareas que lleva a cabo. Adicionalmente, se le asignaron otros atributos derivados de la organización del trabajo en la forma empleo: el trabajador se tenía que construir con referencia tanto a las funciones que se le asignaban para cumplir en un cargo, como con respecto a las habilidades que debía poseer para cumplir adecuadamente con las actividades estipuladas (Sisto, 2009). 
El proyecto psicológico de felicidad que se diseñó para el trabajador sentimental, en la práctica cotidiana intentó traer los valores liberales a los ámbitos laborales bajo el supuesto ético de que la gestión del personal guiada por estos valores traía satisfacción, bienestar y progreso. Se pueden incluir dentro de este proyecto a las psicologías: industrial/organizacional, social de las organizaciones, del trabajo, ocupacional, así como la conducta organizacional, el desarrollo organizacional y la ergonomía entre otras (Guevara, 1989; Hollway, 1991). En estas subáreas se hacen muy diversas combinaciones entre la psicología de las diferencias y la psicología propuesta por la escuela de las relaciones humanas, de manera tal que se actualizan de diversas formas los valores que guían la sociedad liberal (Hollway, 1991; Pulido-Martínez, 2011).

La selección de personal, la capacitación, la evaluación del desempeño, las mediciones de clima organizacional, las estrategias de preparación para el retiro, así como el desarrollo organizacional buscan garantizar, no solamente la satisfacción y la felicidad a través del trabajo, sino que también se convierten en garantes de que los valores de la sociedad liberal se alcancen en la práctica cotidiana de las organizaciones (Pulido-Martínez, 2012). Este proyecto tiene una función importante en las sociedades guiadas por dichos valores liberales. Se supone que las técnicas y estrategias psicológicas al ser aplicadas con la rigurosidad que requieren evitan que aspectos asociados con injusticias o prejuicios sociales hagan su aparición. Es decir, al hacer uso riguroso, por tanto ético, de las estrategias que componen en ciclo psicológico organizacional se impedirá que factores tales como las conexiones de clase, o los prejuicios sociales relacionados con el género, la edad, la preferencia sexual o la raza, sesguen, o mejor, limiten el premio al mérito (Pulido-Martínez, 2010).

Ahora bien, esta visión del trabajo y su derivada propuesta de la subjetividad del trabajador como un ser sentimental, que puede ser fácilmente influido mediante estrategias que buscan incrementar la satisfacción con las actividades y con las relaciones que se establecen en el trabajo, ha sido duramente criticada tanto al interior como al exterior de la disciplina psicológica. Al interior de la psicología, los investigadores han señalado que en la medida en que esta disciplina se ocupa de resolver las preguntas propias de la gerencia sobre ¿cómo administrar la fuerza laboral? se auto-reduce a un instrumento para alcanzar objetivos organizacionales. En esta forma, el conocimiento psicológico no confronta preguntas por el sentido, las cuales asumirían la forma ¿por qué debe ser administrada la fuerza laboral? ¿para quién y por quienes debe ser administrada? Preguntas críticas, o por el sentido de la práctica psicológica, no se toman en consideración puesto que implican un cuestionamiento a las relaciones de poder que se presentan en el trabajo. En estas condiciones, se señala que la psicología solamente se encarga de proveer soluciones técnicas para los problemas que confronta la gerencia. Derivado de esta situación se critica que la psicología, no cuenta, ni encuentra, la necesidad de producir herramientas conceptuales propias para entender el mundo del trabajo (Fernandez-Rios, 1995).

Ante este tipo de señalamientos acerca de los "errores" de la psicología se ha respondido formulando propuestas dirigidas a un constante mejoramiento. Se propone entonces que la psicología debe ser más conceptual para ir más allá de su enfoque pragmático, caracterizado por la producción de obviedades y de la trivialización de los problemas del trabajo (Anderson, Herriot, \& Hodgkinson, 2001; Crespo, Revilla, \& Serrano, 2011). También se ha promovido que se debe configurar una psicología más democrática, en cuanto a que debe abandonar su sesgo pro-gerencial para volverse un saber más autónomo y amplio, disponible para el uso de diferentes grupos con distinto poder relativo.

Se afirma aquí que los psicólogos no deben preocuparse solamente por los intereses de la gerencia, sino que deben tener en consideración los objetivos de los trabajadores, las agremiaciones y los sindicatos (Huzczco, Wiggins, \& Curie, 1984). Se asume que una psicología interesada de modo genuino en asuntos sindicales proveería herramientas para el bienestar general de los diversos grupos que actúan en el mundo del trabajo. El consenso general, en esta línea, indica que si la psicología considerara el interés de otros actores organizacionales que han sido relegados, podría potencialmente tornarse en un saber más igualitario (Rosen \& Stagner, 1980).

Otra propuesta de mejoramiento de esta disciplina considera que debe ser más legal. Se formula entonces que teniendo en cuenta el ejercicio de poder que permite hacer el conocimiento psicológico, se debe reflexionar sobre las intervenciones que se llevan a cabo en los ámbitos laborales, de manera tal que se preserven no solamente los intereses de los negocios, sino que fundamentalmente se garanticen los derechos de los trabajadores. Al plantear que la psicología debe ser más legal se alude a que la legislación de cada país debe ocuparse de vigilar de manera cercana los procesos vinculados con esta en el mundo del trabajo, y no dejarlos a la voluntad o buena disposición de los psicólogos y de los administradores de las organizaciones. 
Al exterior de la disciplina, las críticas de corte marxistas señalan que en el modo de producción capitalista el trabajador no se reconoce en las labores que ejecuta, al punto que en la organización de la producción el trabajador puede fácilmente desconocer cuál será su aporte al producto final. Tanto el proceso como el producto le resultan ajenos al trabajador afirman los psicólogos apoyándose en los trabajos de Marx (Fromm, 1997). No es posible entonces que el ser humano que trabaja dentro del sistema capitalista se reconozca en referencia puesto que no puede considerar el producto como propio. En el proceso productivo el trabajador resulta despojado psíquica y económicamente, los dueños de los medios de producción se apropian del valor relativo a la cantidad de trabajo que el sujeto le imprime a los objetos que se comercializan. En estas condiciones, la figura de la subjetividad que emerge es contraria a la del trabajador resultado del proyecto de felicidad. Surge aquí la figura del trabajador alienado en su interioridad, en las relaciones con los demás y en relación con su pertenencia a la especie (Fromm, 1997).

Los críticos de la disciplina con una orientación marxista, asimismo, argumentan que la psicología no es un saber que pueda conducir a algún tipo de liberación, puesto que deja por fuera de sus propuestas la contradicción central del capitalismo, es decir, niega que exista un problema de clases en los sitios donde se labora. Entonces, la psicología pasa a considerarsele como un saber inocuo, en razón de que no toma en cuenta los problemas de base presentes en las situaciones de trabajo, motivo por el cual, no puede realmente lograr algún tipo de transformación. Braverman (1974), por ejemplo, asigna un lugar ornamental a la psicología del trabajo y sostiene que si la psicología realmente alcanzara alguna de las metas que propone, no sería necesario que se auto-renovara constantemente mediante técnicas nuevas para mejorar la productividad.

En vía contraria, otros críticos de orientación marxista han señalado la peligrosidad de la psicología en relación con el trabajo (Baritz, 1960). Desde su punto de vista, esta disciplina está asociada con una ideología que sirve para ocultar las relaciones de clase subyacentes a la relación de empleo. El argumento corre de la siguiente manera, existe una clase social con el poder para comprar la capacidad de trabajar representado en tiempo de vida, y esta antagoniza con otra clase social que solo cuenta con su tiempo y capacidad para trabajar los cuales deben ser vendidos como mercancías en el mercado.

En medio de esta situación, la psicología actúa como un saber que enceguece a los trabajadores para que no puedan reconocer las relaciones subyacentes a la situación de trabajo, lo cual produce un triple efecto. Primero, problemas políticos de la sociedad presentes en el trabajo se tornan problemas psicológicos que están en cabeza de los trabajadores; segundo, considera que los intereses de los trabajadores son iguales a los intereses de los empleadores; y por último, naturaliza las relaciones sociales de producción al permitir asumir que aquello bueno para los negocios es igualmente bueno para los trabajadores y para la sociedad en general (Baritz, 1960; Prilleltensky, 1994; Wexler, 1983).

En referencia a la primera premisa argumental de la que partió este artículo, hasta aquí, se ha establecido la pertinencia en la manera en que el complejo psi se organizó para comprender e intervenir en las condiciones de organización del trabajo vinculadas con el empleo. Este conocimiento y sus prácticas derivadas proveyeron una serie de formas de concebir al trabajador que resultaban pertinentes y congruentes con las certidumbres laborales y por tanto sociales que el empleo ofrecía. Se ha descrito también como las críticas a la psicologia del trabajo tomaron dos caminos, por una parte señalaron los errores de este conocimiento con el objetivo de formular maneras de mejorarlo, por otra indicaron los horrores de las psicologia con miras a construir una psicologia alternativa que llegara a reemplazar el conocimiento psicológico convencional sobre el trabajo.

\section{La psicología en el post-trabajo}

La segunda premisa argumental que aquí se propone señala que el lugar y las operaciones de la psicología se transforman radicalmente con el cambio en las condiciones laborales asociadas a la incertidumbre. No implican estos cambios que la psicología del trabajo desaparezca, por el contrario esta se intensifica y se transforma asociada a la aparición de nuevos agentes que hacen uso de ella.

Se debe señalar, que el buen el empleo, o el buen trabajo, fue posible en las sociedades del Atlántico Norte que desarrollaron plenamente los postulados del Estado del Bienestar. Para el resto del mundo solamente algunas sectores poblacionales alcanzaron este tipo de organización del trabajo. La formas social empleo se caracterizaba por: conservar un cálculo optimo entre cargas laborales cualitativas y cuantitativas, tener claridad roles, permitir desarrollar las propias habilidades, promover la participación en las decisiones, abrir las posibilidades de nuevos aprendizajes, permitir construir lazos sociales fuertes, adicionalmente, estaba enmarcado en 
un trato justo y democrático, sobre todo, se caracterizaba por ser estable en términos de contratación laboral a largo plazo (Karasek \& Theorell 1990; Lindstrom, 1994; Wainwright \& Calnan, 2002).

Actualmente, a raíz de la implementación de las políticas neoliberales el mundo laboral está cambiando aceleradamente dejando de lado las condiciones anteriormente descritas. Aunque todavía existen grupos de trabajadores que están cobijados por las condiciones de empleo se tiende a que estas se deterioren o que desaparezcan de los escenarios laborales. De este modo, en la actualidad, en los países del Atlántico Norte, la organización del trabajo que tiende a volverse "norma" adquiere características contrarias al empleo. En los países que no forman parte de la órbita de aquellos que se autodenominan desarrollados tienden a desaparecer los ámbitos en donde el empleo se hacía presente, así como se aumentan las condiciones precarias que han prevalecido.

La inestabilidad se hace manifiesta en referencia, entre otros, a aspectos tales como: la ambigüedad en el desempeño de roles, el desdibujamiento o carencia de funciones claramente establecidas, aparición de la pluralidad y disponibilidad para la ejecución de tareas la cual reemplaza el tradicional manual de funciones, el tiempo de la jornada laboral deja de estar estipulado con precisión para pasar a horarios extendidos que no necesariamente conllevan mayor remuneración, los puestos de trabajo pierden también su espacio designado y el trabajador tiene que encontrar su propio lugar para hacer sus actividades, la desaparición del contrato permanente, la negociación del salario en términos de un trabajador a la vez, las relaciones sociales temporales sin mayor significatividad, así como la paulatina disipación de la diferencia entre lo público y lo privado, hoy en día, parecen convertirse en los estándares del trabajo en medio de la geopolítica liberal (Castel, 1997; MoulierBoutang, 2006; Sennett, 2000; Stecher, 2011).

Flexibilización, precarización, desindustrialización, desempleo, tercerización están a la orden del día. Una consecuencia fundamental han traído estos fenómenos para la constitución de la subjetividad laboral. Las actividades como tales, lo que se hace de manera particular el sujeto dentro del aparato productivo, parecen desvanecerse como fuente de producción subjetividad. Si los trabajadores buscan apegarse a los recursos con los que contaban para su construcción como sujetos proporcionadas por el buen trabajo, pueden ser tachados de inflexibles, de tener miedo al cambio, y de no adaptarse a las nuevas demandas que se les formulan desde la gerencia. Contemporáneamente, a los trabajadores se les compele, con intensidad, a reinventarse como requisito para poder alcanzar supuestas ventajas que ofrece el mercado de trabajo.

En esta situación la psicología entonces está encargada, de proveer los conceptos y prácticas para que se haga posible esta reinvención de la subjetividad. No está de mas anotar que la psicología demuestra su plasticidad al poner a disposición del mundo del trabajo una serie de elementos para que el trabajador se pueda concebir sin tener que hacer referencia a un trabajo estable y socialmente reconocido. El trabajador sentimental tiene ahora que ser cualificado con nuevos atributos, ya no puede ser solamente visto con referencia a una interioridad sensible, ahora tiene que ser flexible, dispuesto a enfrentar el cambio, adaptable a las circunstancias, puede y tiene que, adicionalmente, moverse entre tareas de diferente carácter. Se establece en la actual transición en las organizaciones del trabajo, una tensión entre la aparente "pasividad" y "estaticidad" del trabajador del empleo, hacia la permanente actividad del trabajador del post-empleo.

Es necesario precisar aún más, este proceso de recualificación psicológica no va ya dirigido a cambiar las percepciones del trabajador con respecto a las tareas que realiza. El alcance ahora es mucho más amplio, se trata de transformar radicalmente su interioridad para convertirse en un ser propositivo, que abandona su zona de confort y que puede convertirse en protagonista de su propio destino. En este movimiento la distancia que en el empleo prometía acercar la psicología entre la capacidad y la voluntad del trabajador empieza a perder sentido. En su lugar el auto-dominio, la auto-regulación, la auto-estima, la auto-eficacia y la autonomía psicológica reemplazan esta diada.

Consiguientemente, no se requiere de una supervisión externa que vigile la voluntad del trabajador. Se da por hecho en la figura del trabajador flexibilizado que la voluntad ya se ganó al devolverle completamente, en términos de las formas "auto", sus desempeños en el trabajo. La distancia entre voluntad y capacidad parece así desvanecerse al remitirse únicamente a las decisiones propias del trabajador. Se da por descontado que estas decisiones son autónomas en términos referidos a la realización de las tareas, por tanto, se supone que en términos de la ejecución, se producirán tanto el mejor esfuerzo individual posible, así como el más amplio bienestar individual. 
Se tiene entonces que en las condiciones laborales que se están imponiendo conceptos y prácticas de la psicología aplicada en el empleo se reapropian, o transforman, para construir a este trabajador autonomizado. Sin embargo, no se detiene allí las transformaciones en el lugar y las operaciones de la psicología. La conjunción entre psicologia humanista y de las diferencias que constituía las maneras de entender al trabajador en el empleo, ha dado paso a una constelación compuesta por las psicologías socio-construccionistas y positiva con sus concomitantes conexiones de propuestas presentadas en la literatura de auto-ayuda, las cuales ahora tiene una adecuada resonancia con las incertidumbres laborales, de modo tal que contribuyen copiosamente a la creación del sujeto que se requiere.

Por ejemplo, la perspectiva socio construccionista propone que las organizaciones no pueden seguir siendo vistas según la tradición convencional de la administración científica. Esto significa que las empresas no se les pueden seguir considerando como máquinas que cumplen objetivos determinados con estructuras rígidas diseñadas por los administradores. Por el contrario, estas deben ser entendidas como conjuntos de interacciones entre personas que, a la vez, tienen subjetividades fragmentadas por los múltiples escenarios donde efectúan una serie de performances con los cuales obtienen un lugar en el mundo del trabajo. Esta visión contrasta con la visiones que en el empleo proponían una serie de pasos para que el trabajo en una organización permitiera llevar a cabo una carrera, que terminara con el retiro voluntario después de haber ejercido una oficio o profesión en donde podía plasmar una vocación.

Puede decirse que al igual que en la conjunción de psicologías consonante con la forma social empleo, la conjunción pertinente para las condiciones de post-empleo es similar en al menos un aspecto. Las perspectivas construccionistas, las propuestas provenientes de la literatura de autoayuda, la psicología positiva, el coaching y la programación neurolingüística, así como todo el arsenal de estrategias disponibles en el mercado entre los que se cuenta "el secreto", "los siete hábitos de la gente eficaz" y "la culpa la tiene la vaca", tan populares entre los miembros de los departamentos de recursos humanos, para nada cuestionan las ahora condiciones de trabajo. Aun mas, en la actualidad aun la crítica interna se ha hecho menos posible en cuanto la flexibilización, la precarización, la desindustrialización y el desempleo están a la base de la aplicación de sus estrategias psicológicas para la transformación del trabajador.

Vale anotar que no solo se presenta una falta de preocupación por el análisis de las condiciones laborales contemporáneas, acompañando esta carencia, tal vez como compensación, se le dedica mucho espacio a celebrar las posibilidades que se abren con la flexibilización para la construcción de los trabajadores y de las organizaciones. Esto implica un cambio en la concepción del papel del trabajo psicológico en la gestión de las organizaciones. Ahora el conocimiento psicológico, tal como sugieren Gergen y Thatchenkery (1996), se pone en relación con la provisión y administración del simbolismo organizacional, de modo tal que se lleve a cabo el manejo del personal de manera efectiva.

En este orden de ideas, la investigación cualitativa en psicología resulta muy adecuada para las condiciones de flexibilización del trabajo. En la medida en que las negociaciones laborales dejan de ser colectivas y pasan a focalizarse de manera individual en el trabajador, tener un conocimiento particularizado y profundo de ciertos trabajadores resulta una estrategia invaluable a la hora de tomar decisiones para el manejo de la fuerza laboral, y por tanto para la constitución de una subjetividad laboral acorde.

En términos generales, la respuesta que da la disciplina a la transformación del mundo del trabajo, con la introducción de las perspectivas socio-construccionistas y con las propuestas de auto-ayuda de cierta manera se limitan a la tradicional "reactividad" del campo. En estas condiciones, la teorización acerca del mundo del trabajo de nuevo pasa a un segundo lugar, o no se considera importante, y lo que se puede decir es aún más relevante, las áreas de indagación por las cuales se interesa la psicología del trabajo no se amplían; por el contrario, se repiten solamente que ahora utilizando las perspectivas mencionadas. Entonces, en la medida en que no se teoriza sobre el trabajo, sobre las transformaciones que están llevando a la reducción de la forma "empleo permanente", sino que se mejoran las técnicas relacionadas con las áreas tradicionales, por ejemplo, para "re-significarlas", la psicología conserva en lugar de proveedora de técnicas para intervenir la subjetividad, sin embargo la intervención, como se ha hecho notar aquí, ha cambiado su carácter, la extensión de la aplicación y los agentes que la ejecutan.

Con relación a estos puntos vale la pena anotar que se ha consolidado una cultura psicológica que también incluye el mundo del trabajo, en donde los agentes son cada vez menos psicólogos de profesión (Castel, Castel, \& Lovell, 1982). Los gerentes, los entrenadores físicos, los gurús, los consultores, como aquellos que ahora se 
hacen denominar con el apelativo de coach, no necesariamente son psicólogos, pero no por esto hacen menos psicología que aquellos graduados en la disciplina. Todos proponen un trabajo "sobre el yo" referido a "visiones positivas" características del trabajador que se requiere. Sobre estos referentes se propone que es posible llevar a cabo una adecuada conducción de si en el mundo del trabajo actual.

Es decir, ante la desaparición de las certidumbres sociales, se interpela al yo para que se conduzca en un estado de libertad psicológica. Aquí, la figura prominente de la subjetividad es el trabajador autónomo que no requiere de mayores vínculos, que conoce como moverse en el mercado de trabajo y que acumula "capital humano" para explorar, mediante la movilidad entre ámbitos laborales, cuáles son las mejores posibilidades para obtener el mejor rendimiento. "Trabajador portafolio" se le ha llamado a esta figura de la subjetividad que emerge (Sisto, 2009) y que se supone está en capacidad de elegir su propio destino sin "cadenas" que lo aten a un puesto de trabajo. En este contexto, la discusión que se suscita entre aquellos interesados por la gestión del recurso humano alrededor de las competencias laborales, así como el debate acerca de las técnicas cuyo objetivo se dirige facilitar la aparición del sujeto empleable, se constituyen en formas en las cuales la psicología entra en consonancia con las condiciones flexibles de trabajo, y se muestran útiles, y casi indispensables, para la producción del "trabajador portafolio" (Sisto, 2009).

Así como las habilidades y funciones integraban la figura del trabajador sentimental en el empleo, contemporáneamente, tanto competencias como empleabilidad, son conceptos que están dirigidos a complementar una manera de concebir al trabajador portafolio, ahora lleno potencialidades con las cuales debe llegar a convertirse en un empresario de sí mismo. La psicología entonces propone las herramientas para que el trabajador pueda realizar la "venta de si", en un mercado de trabajo cada día más difícil. De esta manera, se favorece la ilusión de que los problemas contemporáneos que afectan al trabajador se deben, en mayor medida, a la ausencia de pro-actividad de los sujetos, a la falta de cultivo de sí mismos, y menos a la incertidumbre de las condiciones laborales.

Alrededor de la propuesta de que el lugar y las operaciones de la psicología contemporánea deben ser estudiados alrededor de las articulaciones del complejo psi (Ingleby, 1985; Rose, 1985), en este artículo se exploraron los cambios que han ocurrido en el mundo del trabajo en referencia al papel que cumple la psicología en el paso del empleo al post-empleo. A este respecto se examinaron estas dos formas de organizar el trabajo alrededor de las figuras de la subjetividad compuestas a través de la psicología. El trabajador sentimental emergió como una manera de entender a los seres humanos en el empleo.

Una cualificación de esta figura en términos de las condiciones de incertidumbre que están buscando imperar ha llevado a la emergencia del trabajador portafolio. En esta transición el papel de la psicología ha variado considerablemente. Por una parte el tipo de psicología que se aplica es diferente, humanista y de las diferencias para el empleo, y de auto-ayuda, de los tipos coaching, programación neurolingüística y socioconstruccionistas para el post-empleo. Estos cambios señalan como maneras en las cuales la psicología se torna en su saber plástico útil para la conducción de los ámbitos laborales en la medida en que pone sus herramientas al servicio de diferente racionalidades para conducir los negocios, y por tanto, para manejar la fuerza laboral.

Queda por tanto cuestionada una vez más la manera en que la psicología se presenta como un saber apolítico. Al querer aparecer como una de las formas en que se manejan los negocios de la mejor forma posible, es decir de manera lógica, eficiente de acuerdo con las disposiciones que la gerencia requiere para el manejo de los asuntos humanos en el trabajo queda completamente involucrada en los asuntos de la política.

Por supuesto, hay que hacer un llamado nuevamente para que todos aquellos involucrados en la cultura psicológica reflexionen sobre las implicaciones que tiene hacer psicología en y para el mundo del trabajo. La construcción de la subjetividad del trabajador no es un asunto vacuo, ni dado por hecho, la psicología y los psicólogos tienen la responsabilidad de examinar las consecuencias de su actuar, más allá de la cuestiones técnicas que les son demandadas.

\section{REFERÊNCIAS}

Anderson, N. H., Herriot, P., \& Hodkinson, G. P. (2001). The practitioner-researcher divide in industrial, work and organizational (IWO) psychology: Where are we now, and where do we go from here? Journal of Occupational and Organizational Psychology, 74(4), 391-411. doi: 10.1348/096317901167451

Baritz, L. (1960). The servants of power: A history of the use of social science in american industry. Middletown, Conneticut: Wesleyan University Press. 
Braverman, H. (1974). Labor and monopoly capital: The degradation of work in the twentieth century. Nova York: Monthly Review Press.

Castel, R. (1997). La metamorfosis de la cuestion social: Una cronica del salariado. Buenos Aires: Paidos.

Castel, R., Castel, F., \& Lovell, A. (1982). The psychiatric society. Nova York: Nova York University Press.

Crespo, E., Revilla, J., \& Serrano, A. (2011). Regulación del trabajo y el gobierno de la subjetividad: La psicologización política del trabajo. In A. Ovejero \& J. Ramos (Coords.), Psicologia social critica (pp. 246-263). Madrid: Editorial Biblioteca Nueva.

Donzelot, J. (1981). Pleasure in work. Ideology \& Consciousness, 9(Winter Issue), 3-28.

Fernandez-Rios, M. (1995). La psicología organizacional en una encrucijada tecnológica y cultural. Psicologia del Trabajo y Organizaciones, 11(3), 49-75.

Fromm, E. (1997). Marx y su concepto del hombre. México: Fondo de Cultura Economica.

Gergen, K. J., \& Thatchenkery, T. J. (1996). Organizational science as social construction: Postmodern potentials. Journal of Applied Behaviroral Science, 32(4), 356-377. doi: 10.1177/0021886396324002

Gillespie, R. (1993). Manufacturing knowledge: A history of the Hawthorne studies. Nova York: Cambridge University Press.

Guevara, C. D. L. (1989). Teorías organizacionales y administrativas: Enfoque crítico. Bogotá: McGraw-Hill.

Hollway, W. (1991). Work psychology and organizational behaviour. Managing the individual at work. Londres: Sage.

Huzczco, G. E., Wiggins, J. G., \& Curie, J. S. (1984). The relations between psychology and organized labor: Past, present and future. American Psychologist, 39(4), 432-440. doi: 10.1037/0003-066X.39.4.432

Ingleby, D. (1985). Professionals as socializers: The "psy complex". Research in Law, Deviance and Social Control, 7, 79-109.

Karasek, R., \& Theorell, T. (1990). Healthy work: Stress, productivity, and the reconstruction of working life. Nova York. Basic Books.

Lindstrom, K. (1994). Psychosocial criteria for good work organization. Scandinavian Journal of Work and Environmental Health, 20(Spec No), 123-133.

Mayo, E. (1990). Problemas sociales de una sociedad industrial. Buenos Aires: Nueva Vision.

Meda, D. (1998). El trabajo un valor en extincion. Buenos Aires: Gedisa.

Moulier-Boutang, Y. (2006). De la esclavitud al trabajo asalariado: Economía histórica del trabajo asalariado embridado. Madrid: Akal.

Prilleltensky, I. (1994). The morals and politics of psychology. Psychological discourse and the status quo. Albany: State University of New York Press.

Pulido-Martínez, H. C. (2010). Autonomia en el trabajo: "El reto por opciones" a la luz de la produccion del sujeto neoliberal. Revista Iberoamericana de Psicología: Ciencia y Teconología, 3(1), 7-15.

Pulido-Martínez, H. C. (2011). Psicología y trabajo: Una relación bajo examen. In B. P. B. Valderrama \& H. Escobar-Melo, Psicología y políticas públicas: Aportes desde la academia (pp. 123-143). Bogotá: Editorial Pontificia Universidad Javeriana.

Pulido-Martínez, H. C. (2012). La investigación sobre la identidad en, para y por el trabajo en América Latina, como ejercicio crítico acerca del mundo laboral. Psykhe, 21(2), 77-85. doi: http://dx.doi.org/10.7764/psykhe.21.2.546

Pulido-Martínez, H. C., \& Carvajal-Marín, L. M. (2014). Pathologization and depathologization of the "free worker" in terms of the psychosocial risk. International Journal of Psychological Research, 7(2), 76-84.

Rose, N. (1985). The psychological complex. Londres: Routledge.

Rose, N. (1996). Inventing ourselves: Psychology, power and regulation. Londres: Sage Publications.

Rose, N. (1999). Governing the soul: The shaping of the private self. Londres: Free Association Books.

Rosen, H., \& Stagner, R. (1980). Industrial/organizational psychology and unions: A viable relationship. Professional psychology, 11(3), 477-483. doi: http://dx.doi.org/10.1037/0735-7028.11.3.477

Sennett, R. (2000). La corrosión del carácter: Las consecuencias personales del trabajo en el nuevo capitalismo. Barcelona: Anagrama.

Sisto, V. (2009). Cambios en el trabajo, identidad e inclusión social en Chile: Desafíos para la investigación. Revista Universum, 24(2), 192-216. doi: http://dx.doi.org/10.4067/S0718-23762009000200011

Standing, G. (2010). Work after globalisation: Building occupational citizenship. Cheltenham: Edward Elgar Publising Limited. 
Stecher, A. (2011). Transformaciones del trabajo y procesos identitarios en el nuevo capitalismo. Notas para discutir el texto lationamericano. In B. Medrano \& W. Galindo, Psicologia social e sues movimentos. 30 anos de ABRAPSO (pp. 207-227). Recife: Editora Universitaria UFPE.

Townley, B. (1994). Reframing human resource management: Power, ethics and the subject at work. Londres: Sage Publications.

Triantafillou, P., \& Moreira, A. (2005). Modern templates of happiness: Performing psychotechniques and spiritualism in Denmark. History of the Human Sciences, 18(2), 87-109. doi: 10.1177/0952695105054183

Viteles, M. (1932). Industrial psychology. Nova York: W.W. Norton y Company.

Walkerdine, V. (2005). Freedom psychology and neoliberal subject. Soundings, 25, 47-61.

Wexler, W. (1983). Critical social psychology. Londres: Routledge.

Wainwrigth, D., \& Calnan, M. (2002). Work stress: The making of a modern epidemy. Londres: Open University Press. 